\title{
A new beta generated Kumaraswamy Marshall-Olkin-G family of distributions with applications
}

\author{
Laba Handique ${ }^{1}$ and Subrata Chakraborty ${ }^{1^{*}}$ \\ ${ }^{1}$ Department of Statistics, Dibrugarh University, Dibrugarh-786004, India \\ Email: handiquelaba@gmail.com \\ *Corresponding Author: subrata_stats@dibru.ac.in \\ Received: $10^{\text {th }}$ April $2017 \quad$ Revised manuscript received: $30^{\text {th }}$ Nov $2017 \quad$ Accepted: $6^{\text {th }}$ Dec 2017
}

\begin{abstract}
Unification of the recently introduced Kumaraswamy Marshall-Olkin-G and Beta Marshall-Olkin-G family of distributions is proposed. A number of important statistical and mathematical properties of the family is investigated. A distribution belonging to the proposed family is shown to perform better than the corresponding distribution from the Kumaraswamy Marshall-Olkin-G and Beta Marshall-Olkin-G family of distributions by considering data fitting with three real life data sets.
\end{abstract}

Key words: Exponentiated family, Power Weighted Moments, AIC and K-S test.

\section{INTRODUCTION}

Efforts to define new families of continuous distributions by extending well-known distributions to provide greater flexibility in modelling different types of data generated from real life situation has received renewed attention of many researchers. To this end several new classes were proposed by adding one or more parameters.

Some important recent contributions in this area include Marshall-Olkin Kumaraswamy-G family introduced by Handique et al., (2017a), Generalized Marshall-Olkin Kumaraswamy-G family (Chakraborty and Handique, 2017a), beta generated Kumaraswamy-G (Handique et al., 2017b), Kumaraswamy Generalized Marshall-Olkin-G family (Chakraborty and Handique, 2017b) and beta Generalized Marshall-Olkin-G family (Handique and Chakraborty, 2016) among others. Alizadeh et al., (2015a) proposed Kumaraswamy Marshall-Olkin-G (KwMO-G) family of distributions as a new extension of the
Marshall-Olkin (MO) family for a given baseline distribution with cumulative distribution function (cdf) $G(t)$, survival function (sf) $\bar{G}(t)=1-G(t) \quad$ and probability density function (pdf) $g(t)$. The cdf and sf of the KwMO- $\mathrm{G}$ are given by

$$
\begin{aligned}
& F^{\mathrm{KwMOG}}(t ; a, b, \alpha) \\
& =1-\left[1-[G(t) /\{1-\bar{\alpha} \bar{G}(t)\}]^{a}\right]^{b} \\
& \text { and } \bar{F}^{\mathrm{KwMOG}_{(}}(t ; a, b, \alpha) \\
& =\left[1-[G(t) /\{1-\bar{\alpha} \bar{G}(t)\}]^{a}\right]^{b}
\end{aligned}
$$

where $\alpha>0(\alpha+\bar{\alpha}=1)$ and $a>0, b>0$ are two additional shape parameters. The density function corresponding to (1) is given by

$$
f^{\mathrm{KwMOG}}(t ; a, b, \alpha)
$$

$=\frac{a b \alpha g(t) G(t)^{a-1}}{[1-\bar{\alpha} \bar{G}(t)]^{a+1}}\left[1-\left\{\frac{G(t)}{1-\bar{\alpha} \bar{G}(t)}\right\}^{a}\right]^{b-1}$

(3) $-\infty<t<\infty ; \alpha>0 ; a>0, b>0$ Alizadeh et al., (2015b) proposed another family of distributions called Beta Marshall-Olkin-G ( $\mathrm{BMO}-\mathrm{G}$ ) family of distributions as a new extension of the Marshall-Olkin (MO) 
family for a given baseline distribution with The cdf, sf and pdf of the BMO-Gare given respectively by

$$
\begin{aligned}
& F^{\mathrm{BMOG}}(t ; m, n, \alpha)=I \frac{G(t)}{1-\bar{\alpha} \bar{G}(t)}(m, n), \\
& \bar{F}^{\mathrm{BMOG}}(t ; m, n, \alpha)=1-I \frac{G(t)}{1-\bar{\alpha} \bar{G}(t)}(m, n) \text { and } \\
& f^{\mathrm{BMOG}}(t ; m, n, \alpha) \\
& =\frac{(1 / B(m, n))\left\{\alpha^{n} g(t) G(t)^{m-1} \bar{G}(t)^{n-1}\right\}}{[1-\bar{\alpha} \bar{G}(t)]^{m+n}}
\end{aligned}
$$

where $B(m, n)=\int_{0}^{1} x^{m-1}(1-x)^{n-1} d x$ and

$I_{t}(m, n)=B(m, n)^{-1} \int_{0}^{t} x^{m-1}(1-x)^{n-1} d x$ denotes

the incomplete beta function ratio. $\alpha>0$ $(\alpha=1-\bar{\alpha})$ and $m>0, n>0$ are two additional shape parameters.

For both these families the authors have studied many statistical and cdf $G(t)$, sf $\quad \bar{G}(t)=1-G(t)$ and pdf $g(t)$. mathematical properties and have shown that distributions belonging to their families provide better fit than some competing models in fitting real data sets.

The main motivation of this article is to provide a new family that unifies KwMO-G and BMO-Gfamilies in to one parent family by using the beta generated technique of Eugene et al. (2002), investigate some of its important statistical and mathematical properties and carry out comparative data modelling applications.

\section{Beta Kumaraswamy Marshall-Olkin-G ( BKwMO - G ) family of distributions}

Here a beta generated KwMO-G (BKwMO-G) family is introduced with pdf, cdf and hazard rate function (hrf) respectively are given by

$$
\begin{aligned}
& f^{\mathrm{BKwMOG}}(t ; m, n, a, b, \alpha) \\
& \begin{array}{c}
\frac{a b \alpha g(t) G(t)^{a-1}}{B(m, n)[1-\bar{\alpha} \bar{G}(t)]^{a+1}}\left[1-\left\{\frac{G(t)}{1-\bar{\alpha} \bar{G}(t)}\right\}^{a}\right]^{b n-1}\left[1-\left[1-\left\{\frac{G(t)}{1-\bar{\alpha} \bar{G}(t)}\right\}^{a}\right]^{b}\right]^{m-1} \\
0<t<\infty, 0<a, b<\infty, m, n>0, \alpha>0
\end{array} \\
& F^{\mathrm{BKwMOG}}(t ; m, n, a, b, \alpha)=I_{1-\left[1-[G(t) /\{1-\bar{\alpha} \bar{G}(t)\}]^{a}\right]^{b}}(m, n)
\end{aligned}
$$

and $h^{\mathrm{BKwMOG}}(t ; m, n, a, b, \alpha)$

$$
\begin{aligned}
=\frac{a b \alpha g(t) G(t)^{a-1}}{B(m, n)[1-\bar{\alpha} \bar{G}(t)]^{a+1}\left[1-I_{1-\left[1-[G(t) /\{1-\bar{\alpha} \bar{G}(t)\}]^{a}\right]^{b}}(m, n)\right]}\left\{1-[G(t) /\{1-\bar{\alpha} \bar{G}(t)\}]^{a}\right\}^{b n-1} \\
\times\left\{1-\left[1-[G(t) /\{1-\bar{\alpha} \bar{G}(t)\}]^{a}\right]^{b}\right\}^{m-1}
\end{aligned}
$$

\section{Important particular cases}

For

(i) $m=n=1$, BKwMO- G $(m, n, a, b, \alpha)$

$\equiv \mathrm{KwMO}-\mathrm{G}(a, b, \alpha)$ (Alizadeh et al.,

2015a), (ii) $a=b=1$,

BKwMO- $\mathrm{G}(m, n, a, b, \alpha) \equiv \mathrm{BMO}-\mathrm{G}(m, n, \alpha)$
(Alizadeh et al., 2015b), (iii) $\alpha=1$, $\operatorname{BKwMO-G}(m, n, a, b, \alpha)=\operatorname{BKw}(m, n, a, b)$

(Handique et al., 2017b).

(iv) $m=n=a=b=1$,

BKwMO-G $(m, n, a, b, \alpha)$

$\equiv \mathrm{MO}(\alpha)$ (Marshall and Olkin, 1997), 
(v) $m=n=\alpha=1, \mathrm{BKwMO}-\mathrm{G}(m, n, a, b, \alpha)$

2011), and (vi) $a=b=\alpha=1$, BKwMO- $\mathrm{G}(m, n, a, b, \alpha) \equiv \mathrm{B}(m, n) \quad$ (Eugene et al., 2002 and Jones, 2004). In the rest of

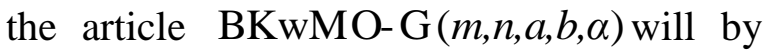
default refer to as BKwMO-G unless specified otherwise.

\section{Genesis of the proposed family of distributions}

Here we present a result to show how this new family may arises as a distribution of order statistics of a sample from KwMO-G $(a, b, \alpha)$ distribution.

Theorem1. If $m$ and $n$ are both integers, then the probability distribution of BKwMO-G $(m, n, a, b, \alpha)$ arises as distribution of $m^{\text {th }}$ order statistics from a random sample size $m+n-1$ from KwMO- $\mathrm{G}(a, b, \alpha)$ distribution.

Proof: Let $T_{1}, T_{2}, \ldots, T_{m+n-1}$ be a random sample of size $m+n-1$ from KwMO-G $(a, b, \alpha)$ distribution with cdf $1-\left[1-[G(t) /\{1-\bar{\alpha} \bar{G}(t)\}]^{a}\right]^{b}$. Then the pdf of the $m^{\text {th }}$ order statistics $T_{(m)}$ is given by

$f^{\mathrm{BKwMOE}}(t ; m, n, a, b, \alpha, \lambda)$

$$
=\frac{a b \alpha \lambda e^{-\lambda t}\left[1-e^{-\lambda t}\right]^{a-1}}{B(m, n)\left[1-\bar{\alpha} e^{-\lambda t}\right]^{a+1}}\left[1-\left\{\frac{1-e^{-\lambda t}}{1-\bar{\alpha} e^{-\lambda t}}\right\}^{a}\right]^{b n-1}\left[1-\left[1-\left\{\frac{1-e^{-\lambda t}}{1-\bar{\alpha} e^{-\lambda t}}\right\}^{a}\right]^{b}\right]^{m-1}
$$

and $h^{\mathrm{BKwMOE}}(t ; m, n, a, b, \alpha, \lambda)$

$$
\begin{gathered}
=\frac{a b \alpha \lambda e^{-\lambda t}\left[1-e^{-\lambda t}\right]^{a-1}}{B(m, n)\left[1-\bar{\alpha} e^{-\lambda t}\right]^{a+1}\left[1-I-\left[1-\left\{\frac{1-e^{-\lambda t}}{1-\bar{\alpha} e^{-\lambda t}}\right\}^{a}\right]^{b}(m, n)\right]}\left[1-\left\{\left(1-e^{-\lambda t}\right) /\left(1-\bar{\alpha} e^{-\lambda t}\right)\right\}^{a}\right]^{b n-1} \\
\times\left[1-\left[1-\left\{\left(1-e^{-\lambda t}\right) /\left(1-\bar{\alpha} e^{-\lambda t}\right)\right\}^{a}\right]^{b}\right]^{m-1}
\end{gathered}
$$

The BKwMO-Weibull (BKwMO-W) distribution $\equiv \mathrm{Kw}-\mathrm{G}(a, b)$ (Cordeiro and de Castro,

$f_{T_{(m)}}(t)=\frac{(m+n-1) !}{(m-1) ![(m+n-1)-m] !}$

$\times\left\{1-\left[1-[G(t) /\{1-\bar{\alpha} \bar{G}(t)\}]^{a}\right]^{b}\right\}^{m-1}$

$\times\left\{\left[1-[G(t) /\{1-\bar{\alpha} \bar{G}(t)\}]^{a}\right]^{b}\right\}^{(m+n-1)-m}$

$\times\left[\left\{\alpha a b g(t) G(t)^{a-1}\right\} /[1-\bar{\alpha} \bar{G}(t)]^{a+1}\right]$

$\times\left\{1-[G(t) /\{1-\bar{\alpha} \bar{G}(t)\}]^{a}\right\}^{b-1}$

$=\left[\left\{a b \alpha g(t) G(t)^{a-1}\right\} /\left\{B(m, n)[1-\bar{\alpha} \bar{G}(t)]^{a+1}\right\}\right]$

$\times\left\{1-[G(t) /\{1-\bar{\alpha} \bar{G}(t)\}]^{a}\right\}^{b n-1}$

$\times\left\{1-\left[1-[G(t) /\{1-\bar{\alpha} \bar{G}(t)\}]^{a}\right]^{b}\right\}^{m-1}$

\section{Plots of the pdf and hrf}

Here we have plotted the pdf and hrf of the BKwMO-G $(m, n, a, b, \alpha)$ taking $\mathrm{G}$ to be exponential (E), Weibull (W), Lomax (L) and Frechet (Fr) distributions for some chosen values of the parameters to show the variety of shapes assumed by the family.

\section{The BKMO-exponential (BKwMO-E) distribution}

When exponential distribution with pdf and cdf $g(t: \lambda)=\lambda e^{-\lambda t}$ and $G(t: \lambda)=1-e^{-\lambda t}$, $t>0, \lambda>0$ is taken as the base line distribution, the pdf and hrf of the resulting BKwMO- Emodel are respectively given by 
$\beta>0$ having pdf and cdf respectively, we get the pdf and hrf of $g(t)=\lambda \beta t^{\beta-1} e^{-\lambda t^{\beta}} \quad$ and $\quad G(t)=1-e^{-\lambda t^{\beta}} \quad$ BKwMO- W distribution as

$f^{\mathrm{BKwMOW}}(t ; m, n, a, b, \alpha, \lambda, \beta)$

$$
=\frac{a b \alpha \lambda \beta t^{\beta-1} e^{-\lambda t^{\beta}}\left[1-e^{-\lambda t^{\beta}}\right]^{a-1}}{B(m, n)\left[1-\bar{\alpha} e^{-\lambda t^{\beta}}\right]^{a+1}}\left[1-\left\{\frac{1-e^{-\lambda t^{\beta}}}{1-\bar{\alpha} e^{-\lambda t^{\beta}}}\right\}^{a}\right]^{b n-1}\left[1-\left[1-\left\{\frac{1-e^{-\lambda t^{\beta}}}{1-\bar{\alpha} e^{-\lambda t^{\beta}}}\right\}^{a}\right]^{b}\right]^{m-1}
$$

and $h^{\mathrm{BKwMOW}}(t ; m, n, a, b, \alpha, \lambda, \beta)$

$$
\begin{gathered}
=\frac{a b \alpha \lambda \beta t^{\beta-1} e^{-\lambda t^{\beta}}\left[1-e^{-\lambda t^{\beta}}\right]^{a-1}}{B(m, n)\left[1-\bar{\alpha} e^{-\lambda t^{\beta}}\right]^{a+1}\left[1-I-\left[1-\left\{\frac{1-e^{-\lambda t^{\beta}}}{1-\bar{\alpha} e^{-\lambda t^{\beta}}}\right\}^{a}\right]^{b}(m, n)\right]}\left[1-\left\{\left(1-e^{-\lambda t^{\beta}}\right) /\left(1-\bar{\alpha} e^{-\lambda t^{\beta}}\right)\right\}^{a}\right]^{b n-1} \\
\times\left[1-\left[1-\left\{\left(1-e^{-\lambda t^{\beta}}\right) /\left(1-\bar{\alpha} e^{-\lambda t^{\beta}}\right)\right\}^{a}\right]^{b}\right]^{m-1}
\end{gathered}
$$

The BKwMO-Lomax (BKwMO-L)

\section{distribution}

Considering the Lomax distribution (Lomax, 1954) with pdf and cdf given by

$$
\begin{aligned}
& g(t: \beta, \delta)=(\beta / \delta)[1+(t / \delta)]^{-(\beta+1)} \text { and } \\
& G(t: \beta, \delta)=1-[1+(t / \delta)]^{-\beta}, \quad t>0, \quad \beta>0,
\end{aligned}
$$

$\delta>0$ the pdf and hrf of the BKwMO-L distribution are given respectively by

$$
\begin{aligned}
& f^{\mathrm{BKwMOL}}(t ; m, n, a, b, \alpha, \beta, \delta) \\
&=\frac{a b \alpha(\beta / \delta)[1+(t / \delta)]^{-(\beta+1)}\left[1-[1+(t / \delta)]^{-\beta}\right]^{a-1}}{B(m, n)\left[1-\bar{\alpha}[1+(t / \delta)]^{-\beta}\right]^{a+1}}\left[1-\left\{\frac{1-[1+(t / \delta)]^{-\beta}}{1-\bar{\alpha}[1+(t / \delta)]^{-\beta}}\right\}^{a}\right]^{b n-1} \\
& \times\left[1-\left[1-\left\{\frac{1-[1+(t / \delta)]^{-\beta}}{1-\bar{\alpha}[1+(t / \delta)]^{-\beta}}\right\}^{a}\right]^{b}\right]^{m-1}
\end{aligned}
$$

and $h^{\mathrm{BKwMOL}}(t ; m, n, a, b, \alpha, \beta, \delta)$

$$
\begin{aligned}
& =\frac{a b \alpha(\beta / \delta)[1+(t / \delta)]^{-(\beta+1)}\left[1-[1+(t / \delta)]^{-\beta}\right]^{a-1}}{B(m, n)\left[1-\bar{\alpha}[1+(t / \delta)]^{-\beta}\right]^{a+1}\left[1-I_{\left.1-\left[1-\left\{\frac{1-[1+(t / \delta)]^{-\beta}}{1-\bar{\alpha}[1+(t / \delta)]^{-\beta}}\right\}^{a}\right]^{b}(m, n)\right]}\right.}\left[1-\left\{\frac{1-[1+(t / \delta)]^{-\beta}}{1-\bar{\alpha}[1+(t / \delta)]^{-\beta}}\right\}^{a}\right]^{b n-1} \\
& \times\left[1-\left[1-\left\{\frac{1-[1+(t / \delta)]^{-\beta}}{1-\bar{\alpha}[1+(t / \delta)]^{-\beta}}\right\}^{a}\right]^{b}\right]^{m-1}
\end{aligned}
$$

The BKwMO-Frechet (BKwMO-Fr)

\section{distribution}

Suppose the base line distribution is the Frechet distribution (Krishna et al., 2013) with pdf and cdf given by $g(t)=\lambda \delta^{\lambda} t^{-(\lambda+1)} e^{-(\delta / t)^{\lambda}}$ and $G(t)=e^{-(\delta / t)^{\lambda}}$, $t>0, \lambda>0, \delta>0$ respectively, then the corresponding pdf and hrf of BKwMO- Fr 
distribution becomes

$$
\begin{aligned}
& f^{\mathrm{BKwMOFr}}(t ; m, n, a, b, \alpha, \lambda, \delta) \\
& =\frac{a b \alpha \lambda \delta^{\lambda} t^{-(\lambda+1)} e^{-(\delta / t)^{\lambda}}\left[e^{-(\delta / t)^{\lambda}}\right]^{a-1}}{B(m, n)\left[1-\bar{\alpha}\left\{1-e^{-(\delta / t)^{\lambda}}\right\}\right]^{a+1}}\left[1-\left\{e^{-(\delta / t)^{\lambda}} /\left[1-\bar{\alpha}\left\{1-e^{-(\delta / t)^{\lambda}}\right\}\right]\right\}^{a}\right]^{b n-1} \\
& \times\left[1-\left[1-\left\{e^{-(\delta / t)^{\lambda}} /\left[1-\bar{\alpha}\left\{1-e^{-(\delta / t)^{\lambda}}\right\}\right]\right\}^{a}\right]^{b}\right]^{m-1}
\end{aligned}
$$

and $h^{\mathrm{BKwMOFr}}(t ; m, n, a, b, \alpha, \lambda, \delta)$

$$
\begin{aligned}
& =\frac{a b \alpha \lambda \delta^{\lambda} t^{-(\lambda+1)} e^{-(\delta / t)^{\lambda}}\left[e^{-(\delta / t)^{\lambda}}\right]^{a-1}}{B(m, n)\left[1-\bar{\alpha}\left\{1-e^{-(\delta / t)^{\lambda}}\right\}\right]^{a+1}\left[1-I-\left[1-\left\{\frac{e^{-(\delta / t)^{\lambda}}}{1-\bar{\alpha}\left\{1-e^{-(\delta / t)^{\lambda}}\right\}}\right\}^{a}\right]^{b}(m, n)\right]} \\
& \times\left[1-\left\{\frac{e^{-(\delta / t)^{\lambda}}}{1-\bar{\alpha}\left\{1-e^{-(\delta / t)^{\lambda}}\right\}}\right\}^{a}\right]^{b n-1}\left[1-\left[1-\left\{\frac{e^{-(\delta / t)^{\lambda}}}{1-\bar{\alpha}\left\{1-e^{-(\delta / t)^{\lambda}}\right\}}\right\}^{a}\right]^{b}\right]^{m-1}
\end{aligned}
$$

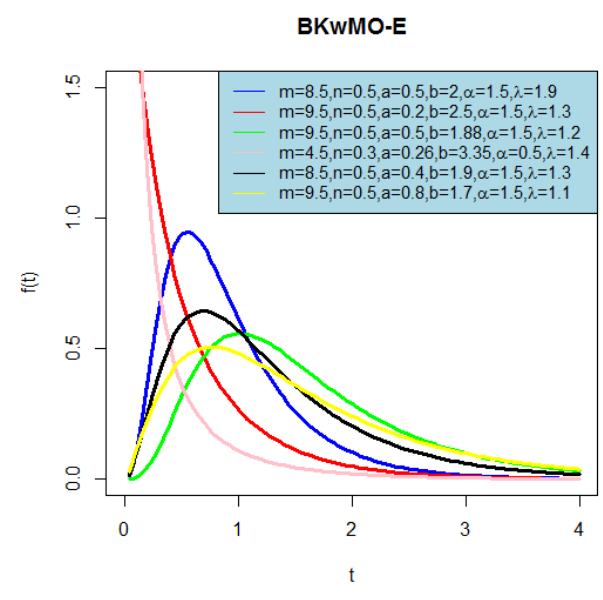

BKWMO-L

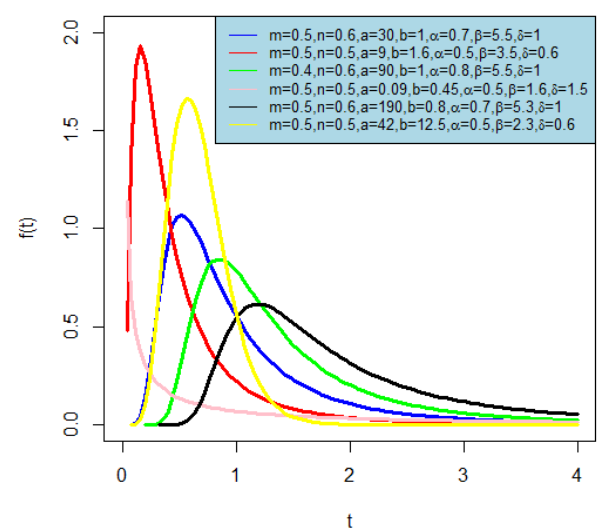

BKwMO-W

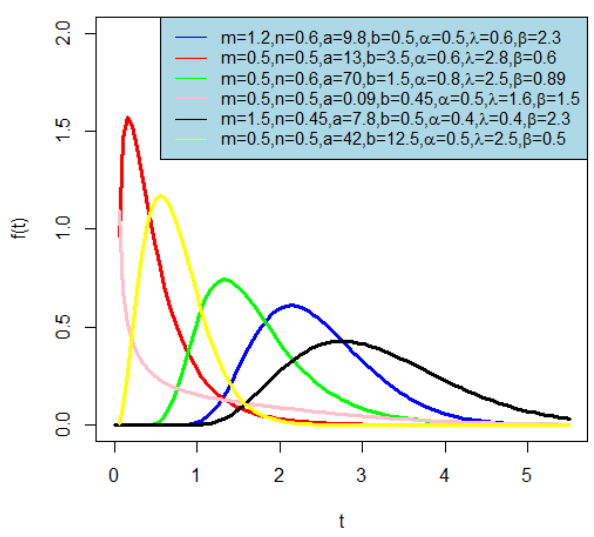

BKwMO-Fr

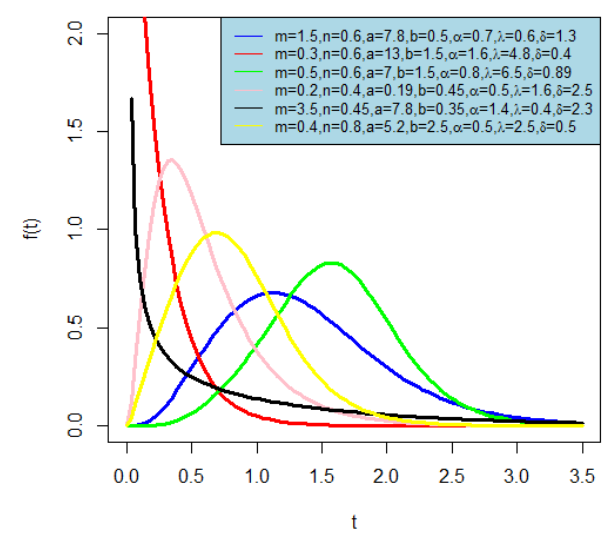

Fig 1 Density plots BKwMO-E, BKwMO- W, BKwMO- Land BKwMO-Fr Distributions clockwise from top left corner. 

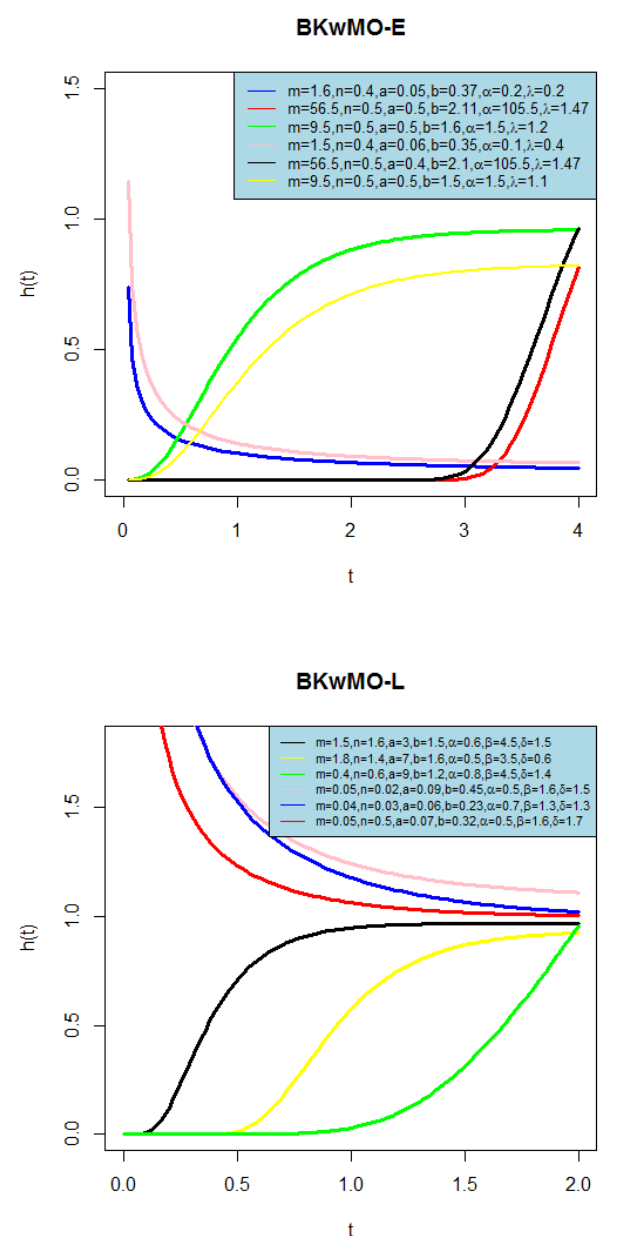
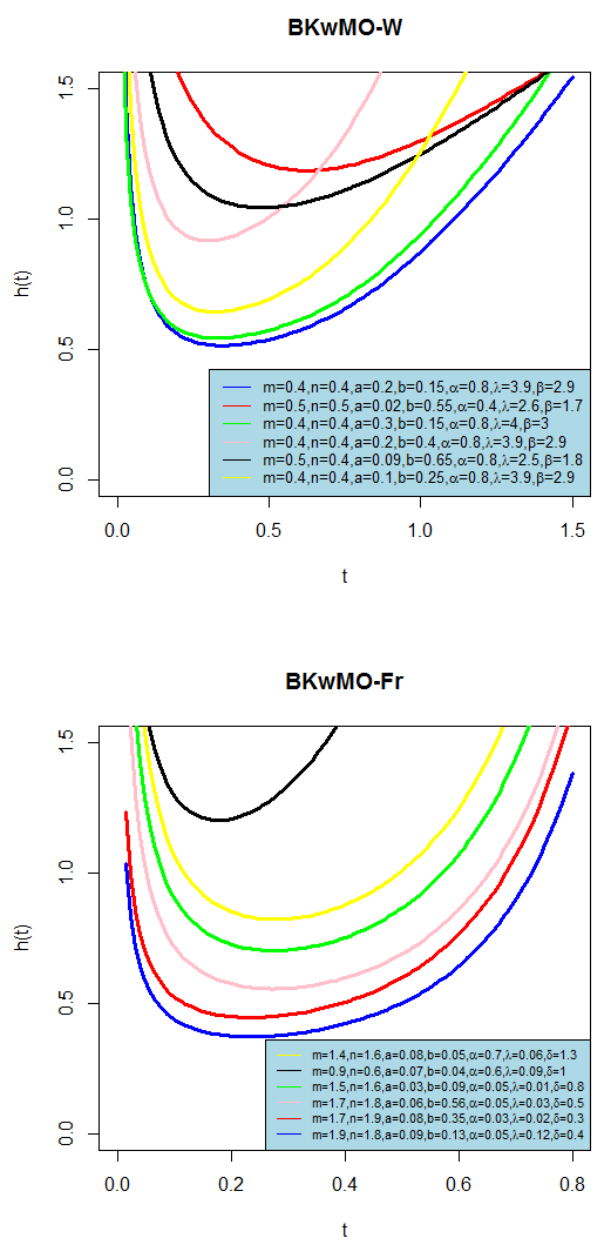

Fig 2 Hazard plots BKwMO-E, BKwMO-W, BKwMO-Land BKwMO-Fr distributions clockwise from top left corner.

From the plots in figure 1 and 2 it can be seen that the family is very flexible and can offer many different types of shapes of density and hazard rate function including the bathtub shape for hazard.

\section{Statistical and mathematical properties}

In this section we derive some general results for the proposed BKwMO- $\mathrm{G}(m, n, a, b, \alpha)$ family.

\section{Series expansions of pdf and cdf}

By using binomial expansion in (4), we obtain

$f^{\mathrm{BKwMOG}}(t ; m, n, a, b, \alpha)$

$$
\begin{aligned}
& =f^{\mathrm{MO}}(t ; \alpha) \sum_{i=0}^{m-1} \sum_{j=0}^{b(i+n)-1} \beta_{i, j} \\
& \times\left[F^{\mathrm{MO}}(t ; \alpha)\right]^{a(j+1)-1} \\
& =\sum_{i=0}^{m-1} \sum_{j=0}^{b(i+n)-1} \beta_{i, j}^{\prime} \frac{d}{d t}\left[F^{\mathrm{MO}}(t ; \alpha)\right]^{a(j+1)}
\end{aligned}
$$

where $\beta_{i, j}^{\prime}=\frac{b}{B(m, n)(j+1)}\left(\begin{array}{c}m-1 \\ i\end{array}\right)$

$$
\times\left(\begin{array}{c}
b(i+n)-1 \\
j
\end{array}\right)(-1)^{i+j},
$$

$\beta_{i, j}=a(j+1) \beta_{i, j}^{\prime}$ and $f^{\mathrm{MO}}(t ; \alpha)$,

$F^{\mathrm{MO}}(t ; \alpha)$ are pdf and cdf respectively of the Marshall-Olkin (MO) family. 
Alternatively, we can expand the pdf as

$$
\begin{aligned}
& f^{\mathrm{BKwMOG}}(t ; m, n, a, b, \alpha) \\
& =f^{M O}(t ; \alpha) \sum_{k=0}^{a(j+1)-1} \gamma_{k}\left[\bar{F}^{\mathrm{MO}}(t ; \alpha)\right]^{k} \\
& =\sum_{k=0}^{a(j+1)-1} \gamma_{k}^{\prime} f^{\mathrm{MO}}(t ; \alpha(k+1))
\end{aligned}
$$

where, $\gamma_{k}^{\prime}=\sum_{i=0}^{m-1} \sum_{j=0}^{b(i+n)-1}(-1)^{k} \beta_{i, j}$

$\times\left(\begin{array}{c}a(j+1)-1 \\ k\end{array}\right)$ and $\gamma_{k}=(k+1) \gamma_{k}^{\prime}$

Similarly an expansion for the cdf of BKwMO-G $(m, n, a, b, \alpha)$ can be derived as

$$
\begin{aligned}
& F^{\mathrm{BKwMOG}}(t ; m, n, a, b, \alpha) \\
& =I_{1-\left[1-\{G(t) / 1-\bar{\alpha} \bar{G}(t)\}^{a}\right]^{b}}(m, n) \\
& =I_{1-\left[1-F^{\mathrm{MO}}(t ; \alpha)^{a}\right]^{b}}(m, n) \\
& =\sum_{i=m}^{m+n-1}\left(\begin{array}{c}
m+n-1 \\
i
\end{array}\right)\left\{1-\left[1-F^{\mathrm{MO}}(t ; \alpha)^{a}\right]^{b}\right\}^{i} \\
& \times\left\{\left[1-F^{\mathrm{MO}}(t ; \alpha)^{a}\right]^{b}\right\}^{m+n-1-i} \\
& =\sum_{i=m}^{m+n-1} \sum_{j=0}^{\infty} \sum_{k=0}^{\infty} \sum_{l=0}^{a k} \eta_{i, j, k, l} \bar{F}^{\mathrm{MO}}(t ; \alpha)^{l}
\end{aligned}
$$

where,

$$
\begin{aligned}
& \eta_{i, j, k, l}=(-1)^{j+k+l}\left(\begin{array}{c}
m+n-1 \\
i
\end{array}\right)\left(\begin{array}{c}
i \\
j
\end{array}\right) \\
& \times\left(\begin{array}{c}
b(m+n-i+j-1) \\
k
\end{array}\right)\left(\begin{array}{c}
a k \\
l
\end{array}\right)
\end{aligned}
$$

Now to expand the cdf $F^{B K w M O G}(t ; m, n, a, b, \alpha)$

$=I_{1-\left[1-[G(t) /\{1-\bar{\alpha} \bar{G}(t)\}]^{a}\right]^{b}}(m, n)$, we use the following result

$$
\begin{aligned}
& I_{z}(a, b)=\frac{B_{z}(a, b)}{B(a, b)} \\
& =\frac{z^{a}}{B(a, b)} \sum_{i=0}^{\infty}\left(\begin{array}{c}
b-1 \\
i
\end{array}\right) \frac{(-1)^{i}}{(a+i)} z^{i}
\end{aligned}
$$

(See "Incomplete Beta Function" From Math World-A Wolfram Web Resource.
http://mathworld.Wolfram.com/Incomplete Beta Function. html).

From (5) using (10) we get

$F^{\mathrm{BKwMOG}}(t ; m, n, a, b, \alpha)$

$=\sum_{i, j, k, p=0}^{\infty} \sum_{l=p}^{\infty} \frac{(-1)^{i+j+k+l+p}}{B(m, n)(m+i)}\left(\begin{array}{c}n-1 \\ i\end{array}\right)\left(\begin{array}{c}m+i \\ j\end{array}\right)$ $\times\left(\begin{array}{l}b j \\ k\end{array}\right)\left(\begin{array}{c}a k \\ l\end{array}\right)\left(\begin{array}{l}l \\ p\end{array}\right)\left[F^{\mathrm{MO}}(t ; \alpha)\right]^{p}$

$=\sum_{p=0}^{\infty} \lambda_{p}\left[F^{\mathrm{MO}}(t ; \alpha)\right]^{p}$

where, $\lambda_{p}=\sum_{i, j, k=0}^{\infty} \sum_{l=p}^{\infty} \frac{(-1)^{i+j+k+l+p}}{B(m, n)(m+i)}\left(\begin{array}{c}n-1 \\ i\end{array}\right)$ $\times\left(\begin{array}{c}m+i \\ j\end{array}\right)\left(\begin{array}{l}b j \\ k\end{array}\right)\left(\begin{array}{c}a k \\ l\end{array}\right)\left(\begin{array}{l}l \\ p\end{array}\right)$

\section{Order statistics}

Suppose $T_{1}, T_{2}, \ldots, T_{\vartheta}$ is a random sample from any BKwMO-G $(m, n, a, b, \alpha)$ distribution. Let $T_{r: 9}$ denote the $r^{\text {th }}$ order statistics. The pdf of $T_{r: \vartheta}$ can be expressed as

$$
\begin{aligned}
f_{r: \vartheta}(t)= & \frac{\vartheta !}{(r-1) !(\vartheta-r) !} \sum_{j=0}^{\vartheta-r}(-1)^{j}\left(\begin{array}{c}
\vartheta-r \\
j
\end{array}\right) \\
& \times f^{\mathrm{BKwMO}}(t) F^{\mathrm{BKwMO}}(t)^{j+r-1}
\end{aligned}
$$

We can now use general expansion of the pdf and cdf in equation last section to get

$$
\begin{aligned}
& f_{r: n}(t)=\frac{\vartheta ! f^{M O}(t ; \alpha)}{(r-1) !(\vartheta-r) !} \sum_{j=0}^{\vartheta-r}(-1)^{j}\left(\begin{array}{c}
\vartheta-r \\
j
\end{array}\right) \\
& \times\left\{\sum_{i=0}^{m-1} \sum_{q=0}^{b(i+n)-1} \beta_{i, q}\left[F^{M O}(t ; \alpha)\right]^{a(q+1)-1}\right\} \\
& \times\left\{\sum_{p=0}^{\infty} d_{j+r-1, p}\left[F^{M O}(t ; \alpha)\right]^{p}\right\} \\
& =\frac{\vartheta ! f}{(r-1) !(\vartheta-r) !} \sum_{j=0}^{M O}(t ; \alpha)
\end{aligned}
$$




$$
\begin{gathered}
\left\{\sum_{q=0}^{b(i+n)-1} \beta_{i, q} d_{j+r-1, p}\left[F^{M O}(t ; \alpha)\right]^{a(q+1)+p-1}\right\} \\
=f^{\mathrm{MO}}(t ; \alpha) \sum_{i=0}^{m-1} \sum_{p=0}^{\infty} \\
\times \sum_{q=0}^{b(i+n)-1} \xi_{i, p, q}\left[F^{\mathrm{MO}}(t ; \alpha)\right]^{a(q+1)+p-1}(12) \\
\text { where, } \xi_{i, p, q}=\frac{\vartheta !}{(r-1) !(\vartheta-r) !} \sum_{j=0}^{\vartheta-r}(-1)^{j} \\
\times\left(\begin{array}{c}
\vartheta-r \\
j
\end{array}\right) \beta_{i, q} d_{j+r-1, p}
\end{gathered}
$$

wherein

$$
d_{j+r-1 p}=\frac{1}{p \lambda_{0}} \sum_{c=1}^{p}[c(j+r)-p] \lambda_{c} d_{j+r-1, p-c}
$$

(Nadarajah et al., 2015) and $\beta_{i, q}, \lambda_{c}$ defined earlier.

\section{Probability weighted moments}

In this section we express probability weighted moments (PWM) of the proposed family in terms of those of $\operatorname{MO}(\alpha)$ distribution.

The $(p, q, r)^{\text {th }}$ PWM of $T$ is defined by $\Gamma_{p, q, r}=\int_{-\infty}^{\infty} t^{p} F(t)^{q}[1-F(t)]^{r} f(t) d t$

(Greenwood et al., 1979). From equations (7) and (9) the $s^{\text {th }}$ moment of $T$ can be obtained either as

$$
\begin{aligned}
& E\left(T^{s}\right)=\int_{0}^{\infty} t^{s} f^{\left.\mathrm{BKwMOG}_{(} t ; m, n, a, b, \alpha\right) d t} \\
& =\sum_{i=0}^{m-1} \sum_{j=0}^{b(i+n)-1} \beta_{i, j} \Gamma_{s, a(j+1)-1,0} \\
& \text { and } E\left(T^{s}\right)=\sum_{k=0}^{a(j+1)-1} \gamma_{k} \int_{0}^{\infty} t^{s}\left[\bar{F}^{\mathrm{MO}}(t ; \alpha)\right]^{k} \\
& =\sum_{k=0}^{\mathrm{MO}}(t ; \alpha) d t
\end{aligned}
$$

where $\Gamma_{p, q, r}$

$=\int_{0}^{\infty} t^{p} F^{\mathrm{MO}}(t ; \alpha)^{q} \bar{F}^{\mathrm{MO}}(t ; \alpha)^{r} f^{\mathrm{MO}}(t ; \alpha) d t$ is the PWM of $\mathrm{MO}(\alpha)$ distribution.

Proceeding similarly we can derive $s^{\text {th }}$ moment of the $r^{\text {th }}$ order statistic $T_{r: \vartheta}$ from a random sample of size $\vartheta$ from BKwMO-G $(m, n, a, b, \alpha)$ using equation (12) as $E\left(T_{r ; \vartheta}^{s}\right)=\sum_{i=0}^{m-1} \sum_{p=0}^{\infty} \sum_{q=0}^{b(i+n)-1} \xi_{i, p, q} \Gamma_{s, a(q+1)+p-1,0}$, where $\beta_{i, j}, \gamma_{k}$ and $\xi_{i, p, q}$ defined earlier.

\section{Generating function}

Here we express the moment generating function of the proposed family in terms of those of the exponentiated $\mathrm{MO}(\alpha)$ distribution using the results of section 3.1 as $M_{T}(s)=\mathrm{E}\left[e^{s T}\right]$

$$
\begin{aligned}
& =\int_{0}^{\infty} e^{s t} f^{\left.\mathrm{BKwMOG}_{(} ; m, n, a, b, \alpha\right) d t} \\
& =\int_{0}^{\infty} e^{s t} \sum_{i=0}^{m-1} \sum_{j=0}^{b(i+n)-1} \beta_{i, j}^{\prime} \frac{d}{d t}\left[F^{\mathrm{MO}}(t ; \alpha)\right]^{a(j+1)} d t
\end{aligned}
$$$$
=\sum_{i=0}^{m-1} \sum_{j=0}^{b(i+n)-1} \beta_{i, j}^{\prime} \int_{0}^{\infty} e^{s t} \frac{d}{d t}\left[F^{\mathrm{MO}}(t ; \alpha)\right]^{a(j+1)} d t
$$$$
=\sum_{i=0}^{m-1} \sum_{j=0}^{b(i+n)-1} \beta_{i, j} M_{X}(s)
$$

where $M_{X}(s)$ is the mgf of an exponentiated (Lehman Alternative-I) $\operatorname{MO}(\alpha)$ distribution.

\section{Rényi entropy}

The entropy of a random variable is a measure of uncertainty variation and has 
been used in various situations in science and engineering. The Rényi entropy of a random variable having pdf $f(t)$ is given by

$$
I_{R}(\tau)=(1-\tau)^{-1} \log \left(\int_{-\infty}^{\infty} f(t)^{\tau} d t\right)
$$

where $\tau>0$ and $\tau \neq 1$. For further details, see Song (2001).

Here

$$
\begin{gathered}
I_{R}(\tau)=(1-\tau)^{-1} \log \left(\sum_{i=0}^{\tau(m-1)} \sum_{j=0}^{b(i+\tau n)-\tau} \eta_{i, j} \int_{0}^{\infty} f^{\mathrm{MO}}(t ; \alpha)^{\tau}\left[F^{\mathrm{MO}}(t ; \alpha)\right]^{a(j+\tau)-\tau} d t\right), \\
\text { where } \eta_{i, j}=\frac{(a b)^{\tau}}{B(m, n)^{\tau}}\left(\begin{array}{c}
\tau(m-1) \\
i
\end{array}\right)\left(\begin{array}{c}
b(i+\tau n)-\tau \\
j
\end{array}\right)(-1)^{i+j}
\end{gathered}
$$

\section{Quantile function, Median and random sample generation}

The quantile function can be obtained by inverting cdf in equation $t=Q(u)$

$=Q_{G}\left(\frac{(1-\alpha)\left[1-\left(1-Q_{m, n}(u)\right)^{1 / b}\right]^{1 / a}}{1-\alpha\left[1-\left[1-\left(1-Q_{m, n}(u)\right)^{1 / b}\right]^{1 / a}\right]}\right)$

where $z=Q_{m, n}(u)$ is the quantile function of beta distribution.

It is possible to obtain some expansions for $Q_{m, n}(u)$ in the Wolfram website (http:// mathworld. wolfram.com/ PowerSeries.html) as $z=Q_{m, n}(u)=\sum_{i=0}^{\infty} e_{i} u^{i / m}$, where $e_{i}=[m B(m, n)]^{1 / m} d_{i}$, $d_{0}=0, d_{1}=1, d_{2}=(n-1) /(m+1)$, $d_{3}=\left\{(n-1)\left(m^{2}+3 m n-m+5 n-4\right)\right\} /$ $\left\{2(m+1)^{2}(m+2)\right\}$ $d_{4}=(n-1)\left[m^{4}+(6 n-1) m^{3}\right.$ $+(n+2)(8 n-5) m^{2}+\left(33 n^{2}-30 n+4\right) m$ $+n(31 n-47)+18] /$ $\left[3(m+1)^{3}(m+2)(m+3)\right]$, etc.

For example, the $p^{\text {th }}$ quantile $t_{p}$ and median of BKwMO-Eare respectively given by

$$
t_{p}=
$$$$
-\frac{1}{\lambda} \log \left[1-\frac{(1-\alpha)\left[1-\left(1-Q_{m, n}(p)\right)^{\frac{1}{b}}\right]^{\frac{1}{a}}}{1-\alpha\left[1-\left[1-\left(1-Q_{m, n}(p)\right)^{\frac{1}{b}}\right]^{\frac{1}{a}}\right]}\right]
$$

and $t_{\text {med }}=$

$$
-\frac{1}{\lambda} \log \left[1-\frac{(1-\alpha)\left[1-\left(1-Q_{m, n}(0.5)\right)^{\frac{1}{b}}\right]^{\frac{1}{a}}}{1-\alpha\left[1-\left[1-\left(1-Q_{m, n}(0.5)\right)^{\frac{1}{b}}\right]^{\frac{1}{a}}\right]}\right] .
$$

Random sample from BKwMO-G can be generated by inverting the cdf.

\section{Skewness and kurtosis}

The Bowley's skewness (Kenney and Keeping, 1962) measures and Moor's kurtosis (Moors, 1988) measure for BKwMO-G family are respectively given in terms of the quantile function as

$$
\begin{aligned}
& B=\frac{Q(3 / 4)+Q(1 / 4)-2 Q(1 / 2)}{Q(3 / 4)-Q(1 / 4)} \\
& M=\frac{Q(3 / 8)-Q(1 / 8)+Q(7 / 8)-Q(5 / 8)}{Q(6 / 8)-Q(2 / 8)}
\end{aligned}
$$

and

\section{Asymptotes}

Some results regarding the asymptotic shapes of the proposed family following is stated here. 
Proposition 1 The asymptotes of pdf, cdf and hrf given in equations (4), (5) and (6) as $t \rightarrow 0$ are given by

$$
\begin{array}{ll}
f(t) \sim\left[\left\{a b g(t) G(t)^{a-1}\right\} /\left\{B(m, n) \alpha^{a}\right\}\right]\left[1-\left\{1-\{G(t) / \alpha\}^{a}\right\}^{b}\right]^{m-1} & \text { as } t \rightarrow 0 \\
F(t) \sim(1 / B(m, n) m)\left[1-\left\{1-\{G(t) / \alpha\}^{a}\right\}^{b}\right]^{m} & \text { as } t \rightarrow 0 \\
h(t) \sim\left[\left\{a b g(t) G(t)^{a-1}\right\} /\left\{B(m, n) \alpha^{a}\right\}\right]\left[1-\left\{1-\{G(t) / \alpha\}^{a}\right\}^{b}\right]^{m-1} & \text { as } t \rightarrow 0
\end{array}
$$

Proposition 2 The asymptotes of pdf, sf and hrf given in equations (4), (5) and (6) as $t \rightarrow \infty$ are given by

$$
\begin{array}{lrl}
f(t) \sim\{(\alpha \operatorname{abg}(t)) / B(m, n)\}\left[1-[1 /\{1-\bar{\alpha} \bar{G}(t)\}]^{a}\right]^{b n-1} & \text { as } & t \rightarrow \infty \\
1-F(t) \sim(1 / n B(m, n))\left[1-[1 /\{1-\bar{\alpha} \bar{G}(t)\}]^{a}\right]^{b n} & \text { as } t \rightarrow \infty \\
h(t) \sim \alpha a b n g(t)\left[1-[1 /\{1-\bar{\alpha} \bar{G}(t)\}]^{a}\right]^{-1} & \text { as } t \rightarrow \infty
\end{array}
$$

\section{Maximum likelihood estimation and data modelling}

The model parameters of the BKwMO-G $(m, n, a, b, \alpha)$ distribution can be estimated by maximum likelihood. Let $t=\left(t_{1}, t_{2}, \ldots, t_{\vartheta}\right)$ be a random sample of size $\vartheta$

$$
\begin{aligned}
\ell=\ell(\boldsymbol{\rho})= & \vartheta \log (a b \alpha)+\sum_{i=1}^{\vartheta} \log \left[g\left(t_{i}, \boldsymbol{\beta}\right)\right]+(a-1) \sum_{i=1}^{\vartheta} \log \left[G\left(t_{i}, \boldsymbol{\beta}\right)\right]-\vartheta \log [B(m, n)] \\
& -(a+1) \sum_{i=1}^{\vartheta} \log \left[1-\bar{\alpha} \bar{G}\left(t_{i}, \boldsymbol{\beta}\right)\right]+(b n-1) \sum_{i=1}^{\vartheta} \log \left[1-\left[G\left(t_{i}, \boldsymbol{\beta}\right) /\left\{1-\bar{\alpha} \bar{G}\left(t_{i}, \boldsymbol{\beta}\right)\right\}\right]^{a}\right] \\
& +(m-1) \sum_{i=1}^{\vartheta} \log \left[1-\left[1-\left[G\left(t_{i}, \boldsymbol{\beta}\right) /\left\{1-\bar{\alpha} \bar{G}\left(t_{i}, \boldsymbol{\beta}\right)\right\}\right]^{a}\right]^{b}\right]
\end{aligned}
$$

This log-likelihood function can not be solved analytically because of its complex form here we have employed global numerical optimization methods to obtain the maximum likelihood estimates (MLEs). By taking the partial derivatives of the log-

$$
\begin{aligned}
& U_{m}=\frac{\partial \ell}{\partial m}=-\vartheta \psi(m)+\vartheta \psi(m+n)+\sum_{i=1}^{\vartheta} \log \left[1-\left[1-\left[G\left(t_{i}, \boldsymbol{\beta}\right) /\left\{1-\bar{\alpha} \bar{G}\left(t_{i}, \boldsymbol{\beta}\right)\right\}\right]^{a}\right]^{b}\right] \\
& U_{n}=\frac{\partial \ell}{\partial n}=-\vartheta \psi(n)+\vartheta \psi(m+n)+b \sum_{i=1}^{\vartheta} \log \left[1-\left[G\left(t_{i}, \boldsymbol{\beta}\right) /\left\{1-\bar{\alpha} \bar{G}\left(t_{i}, \boldsymbol{\beta}\right)\right\}\right]^{a}\right] \\
& U_{a}=\frac{\partial \ell}{\partial a}=\frac{\vartheta}{a}+\sum_{i=1}^{\vartheta} \log \left[G\left(t_{i}, \boldsymbol{\beta}\right)\right]-\sum_{i=1}^{\vartheta} \log \left[1-\bar{\alpha} \bar{G}\left(t_{i}, \boldsymbol{\beta}\right)\right]
\end{aligned}
$$
$m, n, a, b, \alpha$ and $\boldsymbol{\beta}$ components of the score vector $U_{\boldsymbol{\rho}}=\left(U_{m}, U_{n}, U_{a}, U_{b}, U_{\alpha}, U_{\boldsymbol{\beta}^{T}}\right)^{T}$ can be obtained as follows: 


$$
\begin{aligned}
& +(1-b n) \sum_{i=1}^{\vartheta} \frac{\left[G\left(t_{i}, \boldsymbol{\beta}\right) /\left\{1-\bar{\alpha} \bar{G}\left(t_{i}, \boldsymbol{\beta}\right)\right\}\right]^{a} \log \left[G\left(t_{i}, \boldsymbol{\beta}\right) /\left\{1-\bar{\alpha} \bar{G}\left(t_{i}, \boldsymbol{\beta}\right)\right\}\right]}{1-\left[G\left(t_{i}, \boldsymbol{\beta}\right) /\left\{1-\bar{\alpha} \bar{G}\left(t_{i}, \boldsymbol{\beta}\right)\right\}\right]^{a}} \\
& +b(m-1) \sum_{i=1}^{\vartheta} \frac{\left[1-\left[G\left(t_{i}, \boldsymbol{\beta}\right) /\left\{1-\bar{\alpha} \bar{G}\left(t_{i}, \boldsymbol{\beta}\right)\right\}\right]^{a}\right]^{b-1}\left[G\left(t_{i}, \boldsymbol{\beta}\right) /\left\{1-\bar{\alpha} \bar{G}\left(t_{i}, \boldsymbol{\beta}\right)\right\}\right]^{a}}{1-\left[1-\left[G\left(t_{i}, \boldsymbol{\beta}\right) /\left\{1-\bar{\alpha} \bar{G}\left(t_{i}, \boldsymbol{\beta}\right)\right\}\right]^{a}\right]^{b}} \\
& \times \log \left[G\left(t_{i}, \boldsymbol{\beta}\right) /\left\{1-\bar{\alpha} \bar{G}\left(t_{i}, \boldsymbol{\beta}\right)\right\}\right] \\
& U_{b}=\frac{\partial \ell}{\partial b}=\frac{\vartheta}{b}+n \sum_{i=1}^{\vartheta} \log \left[1-\left[G\left(t_{i}, \boldsymbol{\beta}\right) /\left\{1-\bar{\alpha} \bar{G}\left(t_{i}, \boldsymbol{\beta}\right)\right\}\right]^{a}\right] \\
& +(1-m) \sum_{i=1}^{\vartheta} \frac{\left[1-\left[G\left(t_{i}, \boldsymbol{\beta}\right) /\left\{1-\bar{\alpha} \bar{G}\left(t_{i}, \boldsymbol{\beta}\right)\right\}\right]^{a}\right]^{b} \log \left[1-\left[G\left(t_{i}, \boldsymbol{\beta}\right) /\left\{1-\bar{\alpha} \bar{G}\left(t_{i}, \boldsymbol{\beta}\right)\right\}\right]^{a}\right]}{1-\left[1-\left[G\left(t_{i}, \boldsymbol{\beta}\right) /\left\{1-\bar{\alpha} \bar{G}\left(t_{i}, \boldsymbol{\beta}\right)\right\}\right]^{a}\right]^{b}} \\
& U_{\alpha}=\frac{\partial \ell}{\partial \alpha}=\frac{\vartheta}{\alpha}-(a+1) \sum_{i=1}^{\vartheta} \frac{\bar{G}\left(t_{i}, \boldsymbol{\beta}\right)}{1-\bar{\alpha} \bar{G}\left(t_{i}, \boldsymbol{\beta}\right)} \\
& +a(b n-1) \sum_{i=1}^{\vartheta} \frac{G\left(t_{i}, \boldsymbol{\beta}\right)^{a} \bar{G}\left(t_{i}, \boldsymbol{\beta}\right)}{\left[\left\{1-\bar{\alpha} \bar{G}\left(t_{i}, \boldsymbol{\beta}\right)\right\}^{a}-G\left(t_{i}, \boldsymbol{\beta}\right)^{a}\right]\left[1-\bar{\alpha} \bar{G}\left(t_{i}, \boldsymbol{\beta}\right)\right]} \\
& +a b(m-1) \sum_{i=1}^{\vartheta} \frac{\left[\left\{1-\bar{\alpha} \bar{G}\left(t_{i}, \boldsymbol{\beta}\right)\right\}^{a}-G\left(t_{i}, \boldsymbol{\beta}\right)^{a}\right]^{b} G\left(t_{i}, \boldsymbol{\beta}\right)^{a} \bar{G}\left(t_{i}, \boldsymbol{\beta}\right)}{\left[\left\{1-\bar{\alpha} \bar{G}\left(t_{i}, \boldsymbol{\beta}\right)\right\}^{a b}-\left\{\left\{1-\bar{\alpha} \bar{G}\left(t_{i}, \boldsymbol{\beta}\right)\right\}^{a}-G\left(t_{i}, \boldsymbol{\beta}\right)^{a}\right\}^{b}\right]\left[1-\bar{\alpha} \bar{G}\left(t_{i}, \boldsymbol{\beta}\right)\right]} \\
& U_{\boldsymbol{\beta}}=\frac{\partial \ell}{\partial \beta}=\sum_{i=1}^{\vartheta} \frac{g^{(\beta)}\left(t_{i}, \boldsymbol{\beta}\right)}{g\left(t_{i}, \boldsymbol{\beta}\right)}+(a-1) \sum_{i=1}^{\vartheta} \frac{G^{(\beta)}\left(t_{i}, \boldsymbol{\beta}\right)}{G\left(t_{i}, \boldsymbol{\beta}\right)}-(a+1) \sum_{i=1}^{\vartheta} \frac{\bar{\alpha} G^{(\beta)}\left(t_{i}, \boldsymbol{\beta}\right)}{1-\bar{\alpha} \bar{G}\left(t_{i}, \boldsymbol{\beta}\right)} \\
& +a \alpha(1-b n) \sum_{i=1}^{\vartheta} \frac{G\left(t_{i}, \boldsymbol{\beta}\right)^{a-1} G^{(\beta)}\left(t_{i}, \boldsymbol{\beta}\right)}{\left[\left\{1-\bar{\alpha} \bar{G}\left(t_{i}, \boldsymbol{\beta}\right)\right\}^{a}-G\left(t_{i}, \boldsymbol{\beta}\right)^{a}\right]\left[1-\bar{\alpha} \bar{G}\left(t_{i}, \boldsymbol{\beta}\right)\right]} \\
& +a b \alpha(m-1) \sum_{i=1}^{\vartheta} \frac{\left[\left\{1-\bar{\alpha} \bar{G}\left(t_{i}, \boldsymbol{\beta}\right)\right\}^{a}-G\left(t_{i}, \boldsymbol{\beta}\right)^{a}\right]^{b} G\left(t_{i}, \boldsymbol{\beta}\right)^{a-1} G^{(\beta)}\left(t_{i}, \boldsymbol{\beta}\right)}{\left[\left\{1-\bar{\alpha} \bar{G}\left(t_{i}, \boldsymbol{\beta}\right)\right\}^{a b}-\left\{\left\{1-\bar{\alpha} \bar{G}\left(t_{i}, \boldsymbol{\beta}\right)\right\}^{a}-G\left(t_{i}, \boldsymbol{\beta}\right)^{a}\right\}^{b}\right]\left[1-\bar{\alpha} \bar{G}\left(t_{i}, \boldsymbol{\beta}\right)\right]}
\end{aligned}
$$

\section{Asymptotic standard error for the MLEs}

The asymptotic variance-covariance matrix of the MLEs of parameters can obtained by inverting the Fisher information matrix $\mathrm{I}(\boldsymbol{\rho})$ which can be derived using the second partial derivatives of the log-likelihood function with respect to each parameter. The $i j^{\text {th }}$ elements of $\mathrm{I}_{\vartheta}(\boldsymbol{\rho})$ are given by $\mathrm{I}_{i j}=-E\left[\partial^{2} \ell(\boldsymbol{\rho}) / \partial \rho_{i} \partial \rho_{j}\right]$,

$i, j=1,2, \cdots, 5+q$

The exact evaluation of the above expectations may be cumbersome. In practice one can estimate $\mathrm{I}_{\vartheta}(\boldsymbol{\rho})$ by the observed Fisher's information matrix

$$
\begin{aligned}
& \hat{\mathrm{I}}_{\vartheta}(\hat{\boldsymbol{\rho}})=\left(\hat{\mathrm{I}}_{i j}\right) \quad \text { is } \quad \text { defined } \\
& \hat{\mathrm{I}}_{i j} \approx\left(-\partial^{2} \ell(\boldsymbol{\rho}) / \partial \rho_{i} \partial \rho_{j}\right)_{\rho=\hat{\rho}}, \\
& i, j=1,2, \cdots, 5+q
\end{aligned}
$$

Using the general theory of MLEs under some regularity conditions on the parameters as $\vartheta \rightarrow \infty$ the asymptotic distribution of $\sqrt{\vartheta}(\hat{\boldsymbol{\rho}}-\boldsymbol{\rho})$ is $N_{k}\left(0, V_{\vartheta}\right)$, where $V_{\vartheta}=\left(v_{j j}\right)=\mathrm{I}_{\vartheta}^{-1}(\boldsymbol{\rho})$.

The asymptotic behaviour remains valid if $V_{\vartheta}$ is replaced by $\hat{V}_{\vartheta}=\hat{\mathrm{I}}^{-1}(\hat{\boldsymbol{\rho}})$. This result can be used to provide large sample standard errors for the model parameters. Thus an 
approximate standard error for the MLE of $j^{\text {th }}$ parameter $\rho_{j}$ is given by $\sqrt{\hat{v}_{j j}}$.

\section{Comparative data modelling}

\section{Comparison with the KwMO-G and BMO-G}

Here we consider fitting of three real data sets to show that the BKwMO-W distribution from the BKwMO- $W$ family can be a better model than its sub model the KwMO-W and BMO-W (Alizadeh et al., 2015 a, b) distribution.

The density function of the KwMO- $\mathrm{W}$ and BMO- $\mathrm{W}$ are respectively given by

$$
\begin{aligned}
& f^{\mathrm{KwMOW}}(t ; a, b, \alpha, \lambda, \beta) \\
& =\frac{a b \alpha \lambda \beta t^{\beta-1} e^{-\lambda t^{\beta}}\left[1-e^{-\lambda t^{\beta}}\right]^{a-1}}{\left[1-\bar{\alpha} e^{-\lambda t^{\beta}}\right]^{a+1}} \\
& \times\left[1-\left\{\frac{1-e^{-\lambda t^{\beta}}}{1-\bar{\alpha} e^{-\lambda t^{\beta}}}\right\}^{a}\right]^{b-1} \\
& -\infty<t<\infty ; \alpha>0 ; a>0, b>0, \lambda>0, \beta>0 \\
& \text { and } f^{\mathrm{BMOW}}(t ; m, n, \alpha, \lambda, \beta)=
\end{aligned}
$$

$$
\frac{\alpha^{n} \lambda \beta t^{\beta-1} e^{-\lambda t^{\beta}}\left[1-e^{-\lambda t^{\beta}}\right]^{m-1}\left[e^{-\lambda t^{\beta}}\right]^{n-1}}{B(m, n)\left[1-\bar{\alpha} e^{-\lambda t^{\beta}}\right]^{m+n}}
$$

$-\infty<t<\infty ; \alpha>0 ; m>0, n>0, \lambda>0, \beta>0$

We have used AIC, BIC, CAIC and HQIC for model selection, KS test for goodness of fit and Likelihood ratio test for test of hypotheses for nested models.

\section{Likelihood Ratio Test for nested models}

We have seen that the $\mathrm{BKwMO}-\mathrm{W}(m, n, a, b, \alpha ; \lambda, \beta)$ reduces to KwMO-W $(a, b, \alpha ; \lambda, \beta)$, when $m=n=1$ and to BMO-W $(m, n, \alpha ; \lambda, \beta)$ for $a=b=1$, we have therefore used likelihood ratio test to check whether the additional parameter(s) in the proposed model provide statistically significant improvement in data fitting over these nested in sub models.

Here we have employed likelihood ratio criterion to test the following null hypothesis:

$$
H_{0}: m=n=1 \text {, that is the sample is }
$$
from KwMO- $\mathrm{W}(a, b, \alpha, \lambda, \beta)$

$H_{1}: m \neq 1, n \neq 1$, that is the sample is $\mathrm{BKwMO}-\mathrm{W}(m, n, a, b, \alpha, \lambda, \beta)$.

(ii) $H_{0}: a=b=1$, that is the sample is from $\mathrm{BMO}-\mathrm{W}(m, n, \alpha, \lambda, \beta)$

$$
H_{1}: a \neq 1, b \neq 1 \text {, that is the sample is }
$$

$\mathrm{BKwMO}-\mathrm{W}(m, n, a, b, \alpha, \lambda, \beta)$.

Writing $\boldsymbol{\rho}=(m, n, a, b, \alpha, \lambda, \beta)$ the likelihood ratio test statistic is given by $\mathrm{LR}=$ $-2 \ln \left(\ell\left(\hat{\boldsymbol{\rho}}^{*}\right) / \ell(\hat{\boldsymbol{\rho}})\right)$, where $\hat{\boldsymbol{\rho}}^{*}$ is the restricted ML estimates under the null hypothesis $H_{0}$ and $\hat{\boldsymbol{\rho}}$ is the unrestricted ML estimates under the alternative hypothesis $H_{1}$ . Here under the null hypothesis $H_{0}$ the LR criterion follows Chi-square distribution with 2 (two) degrees of freedom (df). The null hypothesis is rejected for $p$-value less than 0.05 .

Data set I: This data set is obtained from Smith and Naynlor (1987). The data consists of 63 observations of the strengths of $1.5 \mathrm{~cm}$ glass fibres, measured at the National Physical Laboratory, England.

Data set II: This data set is a subset of data reported by Bekker et al., (2000) which corresponds to the survival times (in years) of a group of patients given chemotherapy treatment alone. The data consisting of survival times (in years) for 46 patients.

Data set III: This data set about 346 nicotine measurements made from several brands of cigarettes in 1998. The data have been collected by the Federal Trade Commission which is an independent agency 
of the US government, whose main mission is the promotion of consumer protection. [http: //www.ftc.gov/ reports/tobacco or http: // pw1.netcom.com/ rdavis2/ smoke. html.]

The total time on test (TTT) plot proposed by Aarset (1987) is technique helpful in detecting the shape of hazard rate of observed data and hence in deciding a model for fitting. The TTT is drawn by plotting

$T(i / \vartheta)=\left\{\sum_{r=1}^{i} y_{(r)}+(\vartheta-i) y_{(i)}\right\} / \sum_{r=1}^{\vartheta} y_{(r)}$

against $i / \vartheta$,where, $i=1,2, \ldots, \vartheta$ and

$y_{(r)}(r=1,2, \ldots, \vartheta)$ are the order statistics

corresponding to the sample, The hazard of the given data set is constant, decreasing and increasing if the shape of the TTT plot is a straight diagonal line, is of convex shape and concave shape respectively. The TTT plots for the data sets considered here are presented in Fig. 3 which indicates that the data set I and III have increasing hazard rate while for data set II it is nearly constant. We have presented the descriptive statistics of the data sets in Table 1 and findings of the data fittings in Table 2(a) and 2(b).

In Table 2(b), the MLEs, log likelihood, AIC, BIC, CAIC and HQIC values and $\mathrm{KS}, \mathrm{LR}$ statistics are presented for both distributions. According to the lowest values of the AIC, BIC, CAIC and HQIC the BKwMO-W distribution is seen as the better model than both the KwMO-W and BMO-W distribution. Histograms and ogives of the data sets I, II and III along with the fitted pdfs and cdf's are also displayed in Figures 4, 5 and 6 respectively to visually confirm the closeness of the fitted distributions to the observed data. It's easy to see that BKwMO$\mathrm{W}$ provides the best fit to all the data sets considered here.
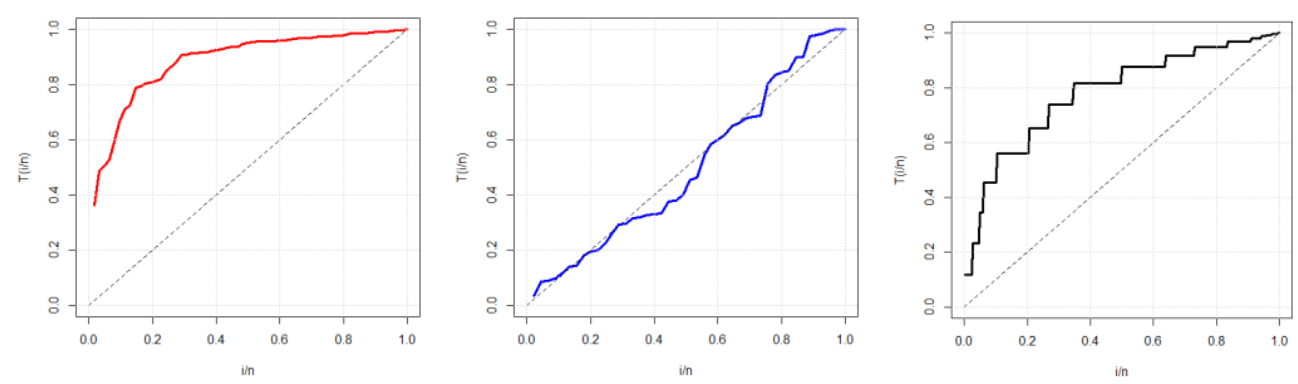

Fig 3: TTT plots of the data sets I, II and III form left to right 
Table 1: Descriptive Statistics for data set I, II and III

\begin{tabular}{cccccccccc}
\hline Data Sets & Minimum & Mean & Median & s.d. & Skewness & Kurtosis & $1^{\text {st }} \mathrm{Qu}$. & $3^{\text {rd }} \mathrm{Qu}$. & Maximum \\
\hline I & 0.550 & 1.507 & 1.590 & 0.324 & -0.879 & 0.800 & 1.375 & 1.685 & 2.240 \\
II & 0.047 & 1.343 & 0.841 & 1.246 & 0.936 & -0.457 & 0.395 & 2.178 & 4.033 \\
III & 0.100 & 0.853 & 0.900 & 0.334 & 0.171 & 0.296 & 0.600 & 1.100 & 2.000 \\
\hline
\end{tabular}

Table 2 (a): MLEs, standard errors (in parentheses) values for data sets I, II and III

\begin{tabular}{lccccccc}
\hline Models & $\hat{m}$ & $\hat{n}$ & $\hat{a}$ & $\hat{b}$ & $\hat{\alpha}$ & $\hat{\lambda}$ & $\hat{\beta}$ \\
\hline Data I & & & & & & & \\
\hline BMO-W & 0.941 & 14.606 & --- & --- & 3.512 & 0.014 & 5.419 \\
$(m, n, \alpha, \lambda, \beta)$ & $(0.302)$ & $(3.659)$ & & & $(1.361)$ & $(0.006)$ & $(0.233)$ \\
KwMO-W & --- & --- & 1.367 & 0.103 & 2.144 & 1.738 & 3.768 \\
$(a, b, \alpha, \lambda, \beta)$ & & & $(0.591)$ & $(0.014)$ & $(0.629)$ & $(0.002)$ & $(0.001)$ \\
BKwMO-W & 0.125 & 0.884 & 4.012 & 0.249 & 12.818 & 0.149 & 6.283 \\
$(m, n, a, b, \alpha, \lambda, \beta)$ & $(0.016)$ & $(0.229)$ & $(1.245)$ & $(0.123)$ & $(5.786)$ & $(0.002)$ & $(2.347)$ \\
Data II & & & & & & & \\
BMO-W & 1.733 & 0.223 & --- & --- & 0.016 & 0.643 & 1.921 \\
$(m, n, \alpha, \lambda, \beta)$ & $(0.325)$ & $(0.089)$ & & & $(0.025)$ & $(0.134)$ & $(0.513)$ \\
KwMO-W & --- & --- & 0.326 & 0.149 & 0.0006 & 0.059 & 3.931 \\
$(a, b, \alpha, \lambda, \beta)$ & & & $(0.142)$ & $(0.032)$ & $(0.001)$ & $(1.581)$ & $(2.367)$ \\
BKwMO-W & 0.093 & 0.081 & 2.288 & 1.499 & 0.0004 & 0.003 & 5.931 \\
$(m, n, a, b, \alpha, \lambda, \beta)$ & $(0.036)$ & $(0.016)$ & $(1.183)$ & $(1.068)$ & $(0.003)$ & $(0.010)$ & $(4.783)$ \\
Data III & & & & & & & \\
\hline BMO-W & 0.237 & 0.917 & --- & --- & 0.014 & 0.005 & 8.571 \\
$(m, n, \alpha, \lambda, \beta)$ & $(0.021)$ & $(0.181)$ & & & $(0.006)$ & $(0.001)$ & $(0.377)$ \\
KwMO-W & --- & --- & 0.438 & 0.883 & 0.124 & 0.110 & 5.470 \\
$(a, b, \alpha, \lambda, \beta)$ & & & $(0.096)$ & $(0.198)$ & $(0.089)$ & $(0.097)$ & $(0.883)$ \\
BKwMO-W & 0.421 & 0.308 & 0.443 & 1.719 & 0.002 & 0.003 & 10.952 \\
$(m, n, a, b, \alpha, \lambda, \beta)$ & $(0.537)$ & $(0.365)$ & $(0.541)$ & $(2.380)$ & $(0.015)$ & $(0.0009)$ & $(0.014)$ \\
\hline & & & & & & & \\
\hline
\end{tabular}


Table 2 (b): AIC, BIC, CAIC, HQIC, KS ( $p$-value) and LR ( $p$-value) values for data sets I, II and III

\begin{tabular}{|c|c|c|c|c|c|c|c|}
\hline Models & $-l_{\text {max }}$ & AIC & $\mathrm{BIC}$ & CAIC & HQIC & $\begin{array}{c}\text { KS } \\
(p \text {-value })\end{array}$ & $\begin{array}{c}\text { LR } \\
(p \text {-value })\end{array}$ \\
\hline \multicolumn{8}{|l|}{ Data I } \\
\hline $\begin{array}{l}\text { BMO-W } \\
(m, n, \alpha, \lambda, \beta)\end{array}$ & 16.43 & 42.86 & 53.56 & 43.91 & 47.06 & $\begin{array}{l}0.08 \\
(0.91)\end{array}$ & $\begin{array}{c}17.66 \\
(0.0001)\end{array}$ \\
\hline $\begin{array}{l}\text { KwMO-W } \\
(a, b, \alpha, \lambda, \beta)\end{array}$ & 19.68 & 49.36 & 60.06 & 50.41 & 53.56 & $\begin{array}{c}0.12 \\
(0.42)\end{array}$ & $\begin{array}{l}24.16 \\
(0.014)\end{array}$ \\
\hline $\begin{array}{l}\text { BKwMO-W } \\
(m, n, a, b, \alpha, \lambda, \beta)\end{array}$ & 7.60 & 29.20 & 44.18 & 31.23 & 35.08 & $\begin{array}{c}0.07 \\
(0.95)\end{array}$ & --- \\
\hline \multicolumn{8}{|l|}{ Data II } \\
\hline $\begin{array}{c}\mathrm{BMO}-\mathrm{W} \\
(m, n, \alpha, \lambda, \beta)\end{array}$ & 56.43 & 122.86 & 132.01 & 124.36 & 126.26 & $\begin{array}{l}0.08 \\
(0.91)\end{array}$ & $\begin{array}{c}8.84 \\
(0.012)\end{array}$ \\
\hline $\begin{array}{l}\text { KwMO-W } \\
(a, b, \alpha, \lambda, \beta)\end{array}$ & 55.97 & 121.94 & 131.09 & 123.44 & 125.34 & $\begin{array}{c}0.58 \\
(0.82)\end{array}$ & $\begin{array}{c}7.92 \\
(0.019)\end{array}$ \\
\hline $\begin{array}{l}\text { BKwMO-W } \\
(m, n, a, b, \alpha, \lambda, \beta)\end{array}$ & 52.01 & 118.02 & 130.83 & 120.97 & 122.78 & $\begin{array}{c}0.54 \\
(0.92)\end{array}$ & --- \\
\hline \multicolumn{8}{|l|}{ Data IIII } \\
\hline $\begin{array}{l}\mathrm{BMO}-\mathrm{W} \\
(m, n, \alpha, \lambda, \beta)\end{array}$ & 109.54 & 229.08 & 248.33 & 229.26 & 236.78 & $\begin{array}{c}0.30 \\
(0.06)\end{array}$ & $\begin{array}{c}7.26 \\
(0.026)\end{array}$ \\
\hline $\begin{array}{l}\text { KwMO-W } \\
(a, b, \alpha, \lambda, \beta)\end{array}$ & 111.49 & 232.98 & 252.23 & 233.15 & 240.68 & $\begin{array}{c}0.26 \\
(0.12)\end{array}$ & $\begin{array}{r}11.16 \\
(0.004)\end{array}$ \\
\hline $\begin{array}{l}\text { BKwMO-W } \\
(m, n, a, b, \alpha, \lambda, \beta)\end{array}$ & 105.91 & 225.82 & 252.77 & 226.15 & 236.60 & $\begin{array}{l}0.25 \\
(0.14)\end{array}$ & --- \\
\hline
\end{tabular}

Estimated pdf's

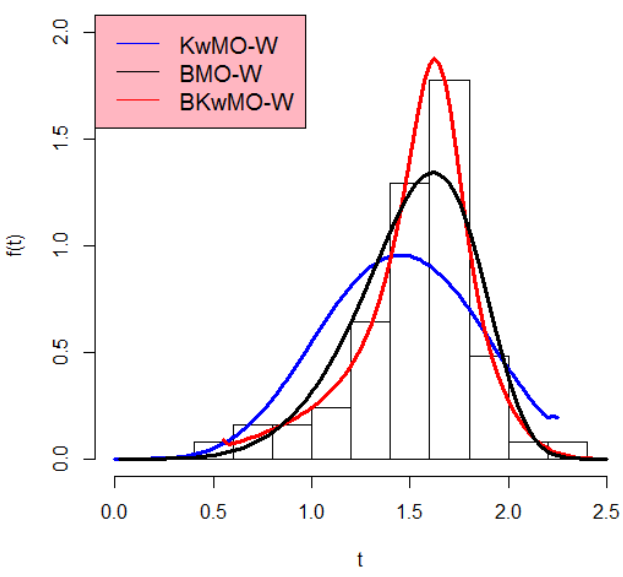

(a)

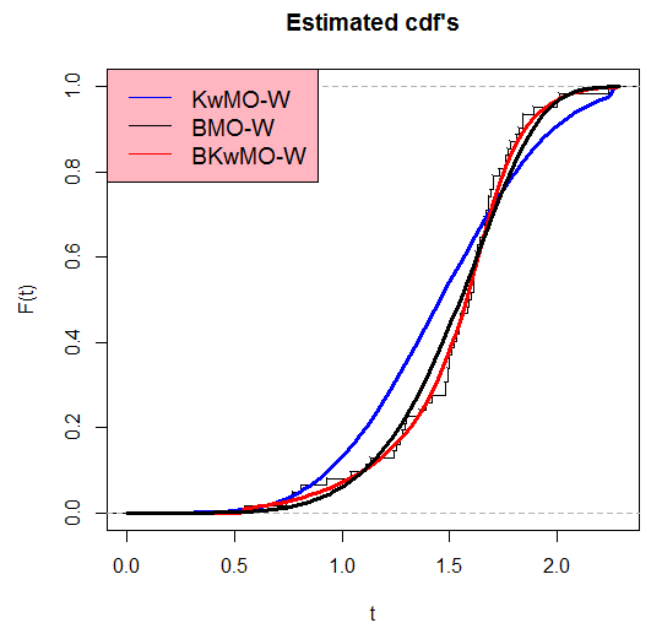

(b)

Fig 4: Plots of the (a) observed histogram and estimated pdf's and (b) observed ogive and estimated cdf's for the KwMO- W, BMO- W and BKwMO- W for data set I. 


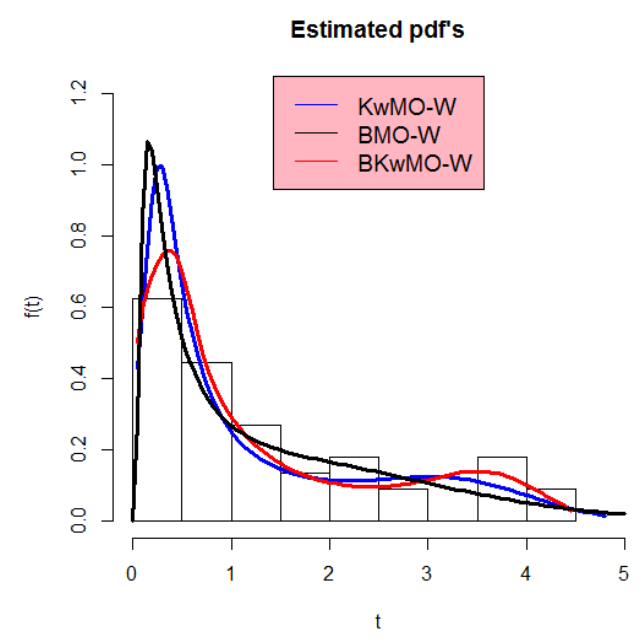

(a)

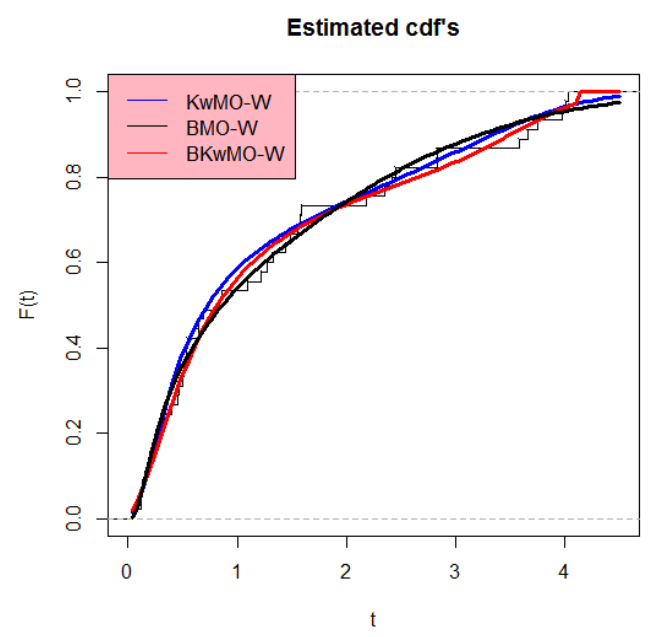

(b)

Fig 5: Plots of the (a) observed histogram and estimated pdf's and (b) observed ogive and estimated cdf's for the KwMO-W, BMO-W and BKwMO- W for data set II.

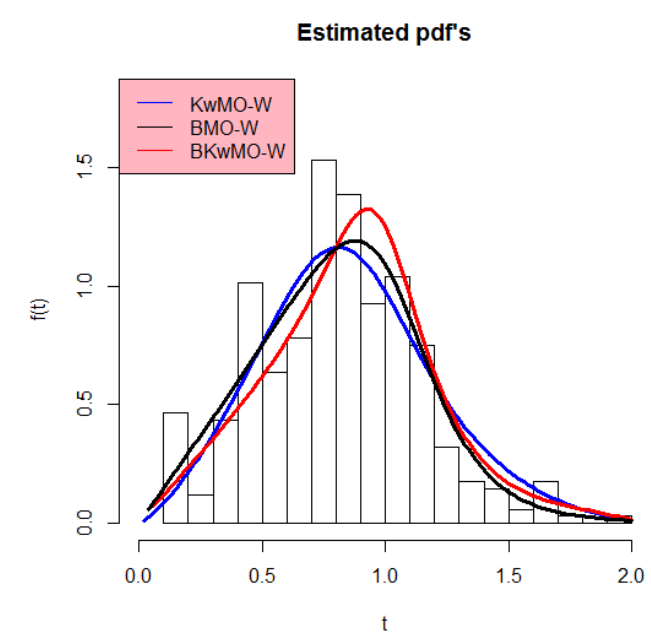

(a)

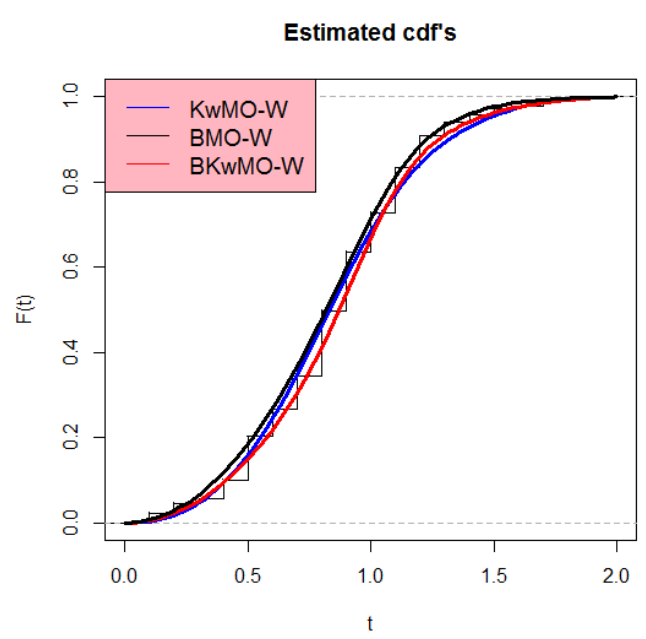

(b)

Fig 6: Plots of the (a) observed histogram and estimated pdf's and (b) observed ogive and estimated cdf's for the KwMO- W , BMO- W and BKwMO- W for data set III.

From the findings tabulated in Table 2(b), on the basis of AIC, BIC, CAIC, HQIC and K-S test it is evident that the $\mathrm{BKwMO}-\mathrm{W}$ distribution is a better model than both the KwMO-W and $\mathrm{BMO}-\mathrm{W}$ for all the data sets considered here. The plots of observed and the estimated pdfs and cdfs also support the same findings. The likelihood ratio test has favored the BKwMO- $\mathrm{W}$ in four out of the six comparisons considered here.

\section{CONCLUSION}

A new Beta Kumaraswamy Marshall-Olkin family of distributions is introduced and 
some of its important properties are studied. The maximum likelihood method for estimating the parameters is implemented. Three applications of real life data fitting shows encouraging result in favour of the proposed unified family over Kumaraswamy Marshall-Olkin and the Beta Marshall-Olkin family of distributions.

\section{ACKNOWLEDGMENT}

Authors would like to acknowledge the critical comments and observations of the reviewers which have helped in improving the presentation.

\section{REFERENCES}

Alizadeh, M., Tahir, M.H., Cordeiro, G.M., Zubair, M. \& Hamedani, G.G. (2015a). The beta Marshall-Olkin family of distributions. Journal of Statistical Distributions and Applications 2:4 doi:10.1186/s40488-015-0027-7.

Alizadeh, M., Tahir M.H., Cordeiro G.M., Zubair M. \& Hamedani G.G. (2015b). The Kumaraswamy Marshal-Olkin family of distributions. Journal of the Egyptian Mathematical Society 23: 546-557.

Aarset, M.V. (1987). How to identify bathtub hazard rate. IEEE Transactions on Reliability 36: 106-108.

Bekker, A., Roux, J. \& Mostert, P. (2000). A generalization of the compound Rayleigh distribution: using a Bayesian methods on cancer survival times. Commun. Stat. Theory Methods 29: 1419-1433.
Chakraborty, S. \& Handique, L. (2017a). The Generalized Marshall-OlkinKumaraswamy-G family of distributions. Journal of Data Science 15: 391-422.

Chakraborty, S. \& Handique, L. (2017b). Properties and data modeling applications of the KumaraswamyGeneralized Marshall-Olkin-G family of distributions. (under review).

Cordeiro, G.M. \& De Castro, M. (2011). A new family of generalized distributions. J Stat Comput Simul 81: 883-893.

Eugene, N., Lee, C. \& Famoye, F. (2002). Beta-normal distribution and its applications. Commun. Stat. Theory Methods 3: 497-512.

Greenwood, J.A., Landwehr, J.M., Matalas, N.C. \& Wallis, J.R. (1979). Probability weighted moments: definition and relation to parameters of several distributions expressible in inverse form. Water Resour Res 15:1049-1054.

Handique, L. \& Chakraborty S. (2016). The Beta Generalized MarshallOlkin-G family of distributions. arXiv:1608.05985 V1 [math.ST]. (under review)

Handique, L., Chakraborty, S. \& Hamedani, G.G. (2017a). The Marshall-Olkin-Kumaraswamy-G family of distributions. Journal of Statistical Theory and Applications (forthcoming, accepted for publication).

Handique, L., Chakraborty, S. \& Ali, M.M. (2017b). The Beta-Kumaraswamy$\mathrm{G}$ family of distributions. Pakistan Journal of Statistics 33: 467-490. 
Jones, M.C. (2004). Families of distributions arising from the distributions of order statistics. Test 13: $1-43$.

Kenney, J.F. \& Keeping, E.S. (1962). Mathematics of Statistics, Part 1. Van Nostrand, New Jersey, 3rd edition.

Krishna, E., Jose, K.K. \& Ristić, M. (2013). Applications of MarshallOlkin Fréchet distribution. Comm. Stat. Simulat. Comput 42: 76-89.

Lomax, K.S. (1954). Business Failures; Another example of the analysis of failure data. Journal of the American Statistical Association 49: 847-852.

Marshall, A. \& Olkin, I. (1997). A new method for adding a parameter to a family of distributions with applications to the exponential and Weibull families. Biometrika 84: 641-652.
Moors, J.J.A. (1988): A quantile alternative for kurtosis. The Statistician 37: 25-32.

Nadarajah, S., Cordeiro, G.M. \& Ortega, E.M.M. (2015). The ZografosBalakrishnan-G family of distributions:mathematical

properties and applications. Commun. Stat. Theory Methods 44: 186-215.

Smith, R.L. \& Naylor, J.C. (1987). A comparison of maximum likelihood and Bayesian estimators for the three-parameter Weibull distribution. Applied Statistics 36: 358-369.

Song, K.S. (2001). Rényi information, loglikelihood and an intrinsic distribution measure. Journal of Statistical Planning and Inference 93:51-69.

Weibull, W. (1951). A statistical distribution function of wide applicability. J. Appl. Mech.-Trans. ASME, 18: 293-297. 